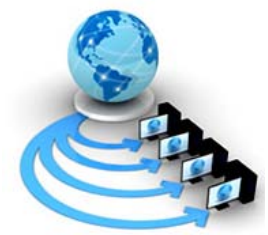

Volume 9, No. 3, May-June 2018

International Journal of Advanced Research in Computer Science

RESEARCH PAPER

Available Online at www.ijarcs.info

\title{
DETECTING SINKHOLE ATTACK IN MANET USING OLSR ROUTING PROTOCOL WITH ARTIFICIAL INTELLIGENCE
}

\author{
Iqbal Singh \\ M.Tech Scholar (CSE Deptt.) \\ SBBSIET, Padhiana,India
}

\author{
Harpreet kaur \\ Assistant Prof. (CSE Dept \\ SBBSIET, Padhiana,India
}

\begin{abstract}
Security is known as the necessity in mobile ad hoc network for the security of the private information. The routing protocols are the major reasons for the insecurity of the information as they take the information by providing the inaccurate route. From all the attacks, sink hole is the one that occur vigorously in the network. In this attack, the thief hops send wrong message of routing to consider itself as a defined hop and make it believed that the network will itself send the information. When the whole network is entertained, it changes the secretive information like change of data packer and developing the network more composite by packet dropping. This research has utilized OLSR routing protocol for discovering the route among source as well as destination in the network defined. For the enhancement of the research, ABC algorithm has been used along with OLSR routing protocol. ABC is the algorithm which is used for the optimization of the route with the selection process. Varied parameters, viz. Throughput, MSE (mean square error), delay, BER (bit error rate) with energy consumption are computed to check the performance of the work in MATLAB simulation tool
\end{abstract}

Keyword: MANET, Proactive link state routing protocol (OLSR), artificial bee colony (ABC) , Artificial neural network (ANN) and MATLAB.

\section{INTRODUCTION}

MANET (Mobile ad hoc network) is self deliberated network that groups different connection less routers or nodes [1]. MANET doesn't have any centralized administration or infrastructure. The routers in MANET can move randomly and freely and later manages themselves. Therefore, the topology can vary very fast and suddenly in wireless network. MANET plays a significant role in dynamic nature. It is known as one of the substantial factors for performance. It permits the mobile needs to go away or link up from the network liberally. When the other wireless choices are been compared, the wireless ad hoc network gives random dynamic topology [2]. The ability of mobile nodes communication is not inadequate. In the network, the mobile nodes may simply mobile in the network area. If the connection has been developed already in ad hoc network and the mobile node can be out of reach of the radio range at the time when the loss of data can be takes place while transmission [3].

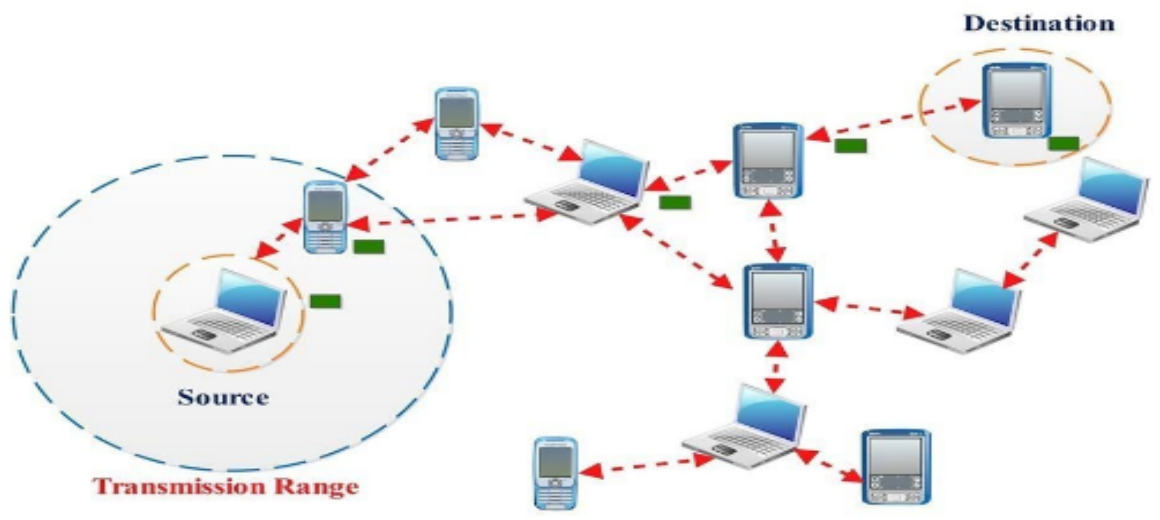

Figure 1: MANET (Mobile Ad hoc network)

The security issue has turn out to be general concern for mobile network for securing the communication among mobile hops in an aggressive network [4]. The intruder and the validate network can execute the wireless channel. The attacks can be classifies into active and passive attacks. The passive attacks can change some data, but attention should be paid to the network. But the active attack can insert the data within the network area like alteration, duplication and elimination of exchanging data and so on [5]. The ad hoc network is susceptible for some certain attacks. Therefore, sink hole attack can be considered as one of the risky attacks in MANET. There are more than 20 routing algorithms; still the Ad hoc Network suffers the coverage, presence of intruders and cost management problems in routing. The first problem of this research work is apply a trust model which would prevent the system from any kind of extra cost in search of suitable node for the transfer of the data [6]. For such purpose, no algorithm other than optimization based algorithm can be thought of. Here the purpose would be solved using Artificial Bee Colony (ABC) with OLSR routing protocol. OLSR is an IP routing protocol optimized for mobile Ad hoc networks using ABC algorithm. Another 
face of Ad hoc Network is that the network has always struggled in managing the intrusion. With the passage of time, attackers have become smarter and in the similar fusion, smart attack, namely, Sink Hole has come into light [7]. The attacks have been termed "SMART" due to their unpredictable behaviour in the network. It has been seen often that the network does not even come to know that it is under the influence of any attack if smart attacks are considered. There is one more issue which should be focused and that would be prevention of innocent nodes, getting harmed due to suspicion [8]. An intelligent sense of think is required in such situation which can take dynamic decision. Artificial Intelligence can be a suited solution for this problem and hence, the problem statement of this research work includes the introduction of neural network technique with the appropriate rule sets [9]. The evaluation of the solution would be based on QOS (Quality of Service) Parameters. The expected parameters would be; Throughput; Error Rate; Energy Consumption; Delay and Mean Square error [10].

\section{MATERIALS AND METHODS}

This research has developed a novel algorithm for the detection and mitigation of sinkhole attack using $\mathrm{NN}$ (Neural networks) with ABC (artificial bee colony algorithm).

\subsection{NN (Neural Network) algorithm}

A Neural Network Classifier is based on neural networks consisting of interconnected neurons. From a simplified perspective, a neuron takes positive and negative stimuli (numerical values) from other neurons and when the weighted sum of the stimuli is greater than a given threshold value, it activates itself. The output value of the neuron is usually a non-linear transformation of the sum of stimuli [11]. Neural network can be trained to perform a particular function by adjusting the values of the weights between elements [12]. Network function is determined by the connections between elements. There is activation functions used to produce relevant output.

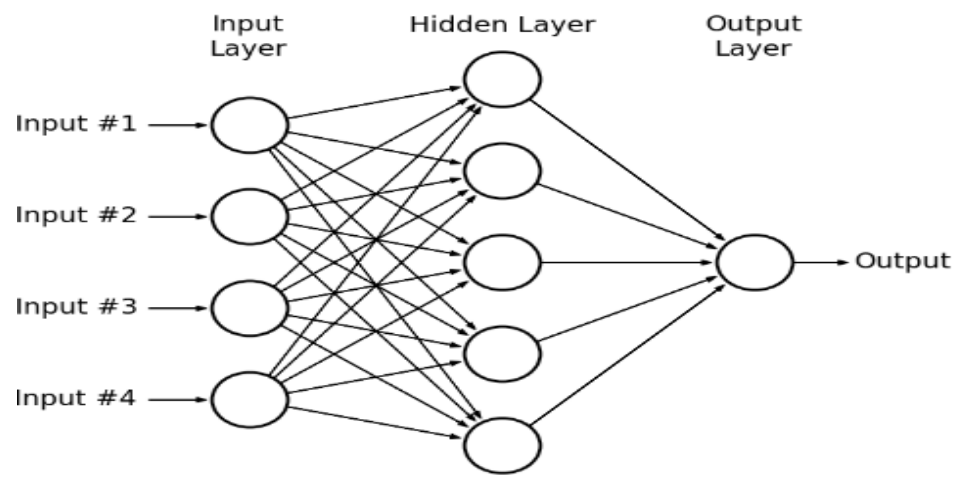

Figure 2: Neural network

The various advantages of using neural network are given below:

- Unlike rule based systems or programmed systems neural networks are flexible in changing environment. Rule based systems are limited to the situation for which they have been designed. If the situation changes they are unable to operate in changed environment. Though neural may take time to learn to a sudden change, they are good at adapting to changing situations

- $\quad$ Since the system is developed through learning and not by programming, neural nets teach themselves the pattern. It can be implemented in any application and without any problem.

- Patter recognition is known as an efficient method for the connection of information in the data with the generalization procedure. $\mathrm{NN}$ recognizes the pattern within the dataset.

- It develops the system that depicts the data structure in less time span.

- It may develop the information system easily as it may handle more composite circumstances than traditional work.

- NN executes accurately with novel computer systems.
- $\quad \mathrm{NN}$ can maintain without some issue in the failing of some elements.

\subsection{ABC (Artificial neural network) algorithm}

$\mathrm{ABC}$ is known as an optimization algorithm that gives procedure of population dependent with the individuals calls the food positions being modified from artificial bees in time [13]. The aim of the bees is to develop the food source places with more nectar quantity and the one with more nectar. In this algorithm, the bee's flies within the space of multi-dimensional search and with few of onlooker and employed bees that select the food sources that depends on its skills and nest mates skills. Few of the scouts select the food sources arbitrarily with no skills. When the nectar amount of novel source is more as compared to the traditional one than they remember the novel positions and overlooks the existing one. Therefore, it integrates the local search techniques and executes the onlooker and employed bees with methods that are assisted by scouts and onlooker bees and tries to balance the exploitation and exploration procedure [14].

The essential ABC algorithm can be divided into three stages:
i. Employed bee phase
ii. Onlooker bee phase
iii. Scout bee phase 
A bee waiting on the dance area for making decision to choose a food source is called an onlooker and a bee going to the food source visited by itself previously is named an employed bee. A bee carrying out random search is called a scout. Colonies initial half is consisted of employed artificial bees and the subsequent part has onlookers. Single employed bee is always there for food sources. It can also be said that the amount of employed bees is proportional to the amount of food sources in the hive. Scout develops when the employed bees has exhaustive food sources from the onlooker and the employed bees [15].

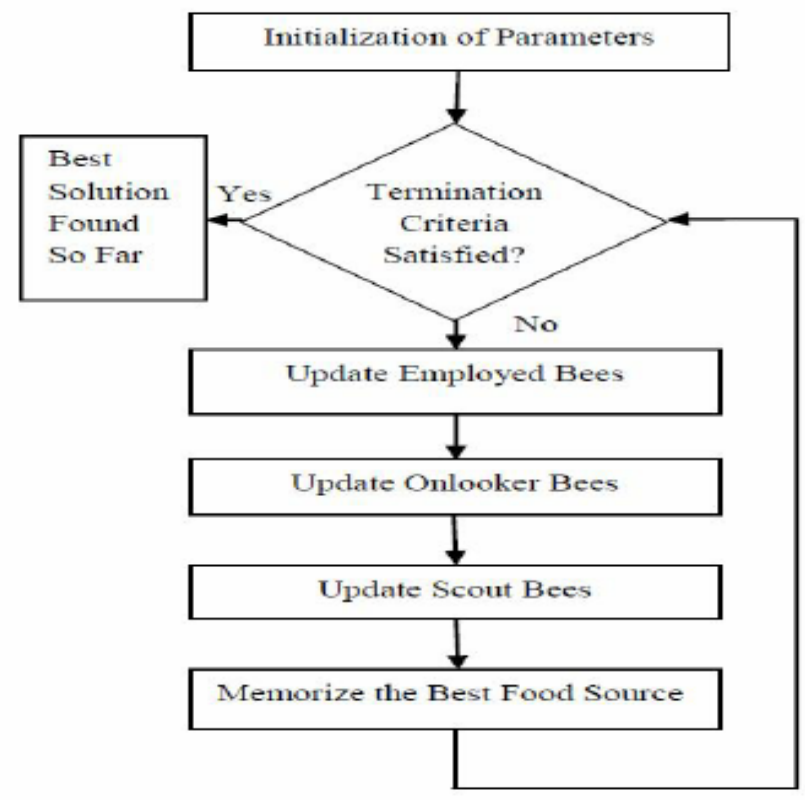

Figure 3: ABC working flowchart

\section{PROPOSED ARCHITECTURE}

In this research, $A B C$ algorithm is utilized for optimization along with OLSR routing protocol. ANN is used for the classification of the proposed work. The utilization of these two is for the reduction of the issue of routing protocols and decrement of the energy usage. Numbers of steps are taken while executing the model as shown and defined below.

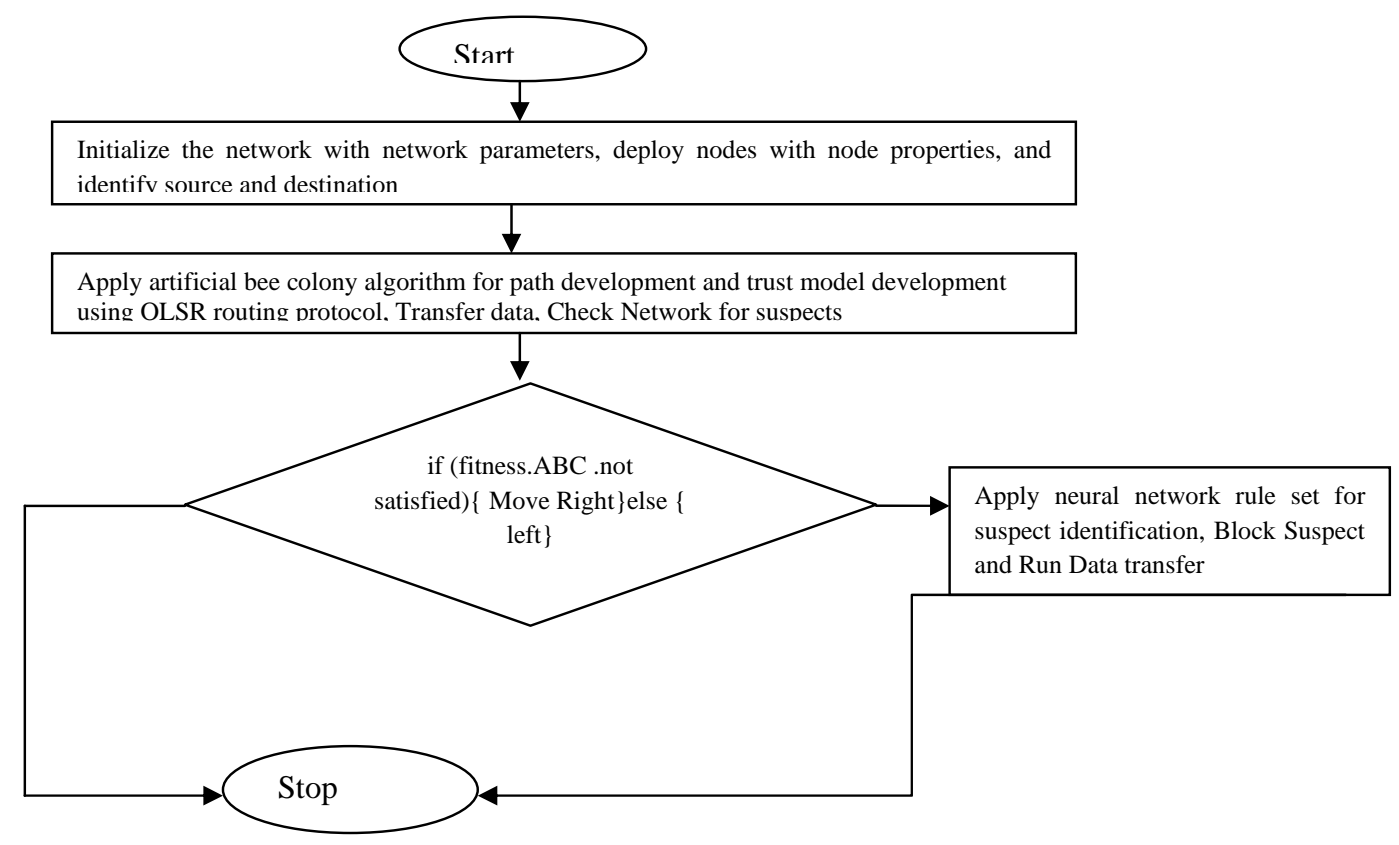

Figure 4: Methodology of proposed work 
I. To develop a network of MANET by means of height and width of the network

II. Initiate the $\mathrm{N}$ amount of nodes in the network.

III. Describe the source node as well as destination node in terms of initiative

IV. Arrange the coverage area for every node and arrange the numeral of round for executing the network.

V. Develop a route initiated from source and preceded towards destination from utilized OLSR routing protocol.

VI. Locate the objective function as per the requirements. The formula for the same is defined below:

Initialize artificial bee colony algorithm to optimize route and achieve the better throughput rate using the novel fitness function. The fitness function of proposed work is shown below

\section{Fit}

Where $F_{g}$ is the selected properties of the individual nodes and the $F_{t}$ is the threshold properties and it is the average of all nodes properties.

VII. For the optimization of the path, ABC algorithm is used that discovers the effective route for the transmission of the packets.

VIII. If there is no satisfaction of the objective function than use ANN and apply the rule set for finding the suspected nodes. Then, the transmission of data stared.

\section{RESULTS AND DISCUSSION}

The results being obtained after the simulation of the proposed work are described in this section. The diagrams shown below define the environment considered for the simulation which has number of nodes as $50.1000 * 1000$ is the width and the height taken to run the system for around five rounds.

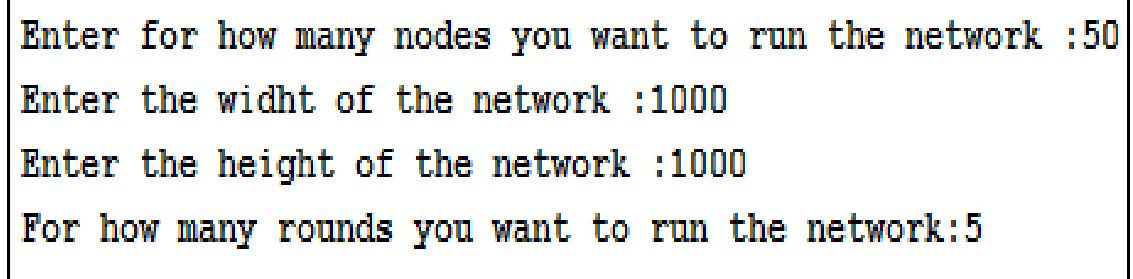

Figure Error! No text of specified style in document.: Simulation Environment

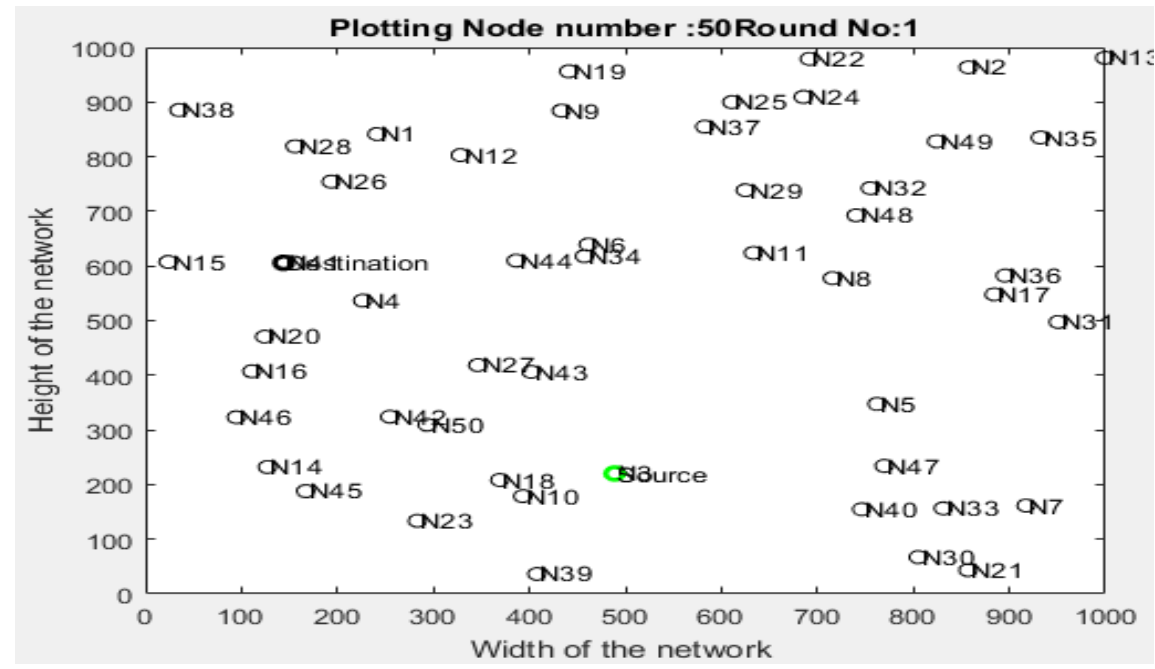

Figure 6: Simulation Window

Above figure is showing the simulation window for 50 nodes within the network. The network is being created for fixed height and width. The green point is for the source and the black is for the destination. The X-axis is describing the width of the network whereas the Y-axis is describing the height of the network. 


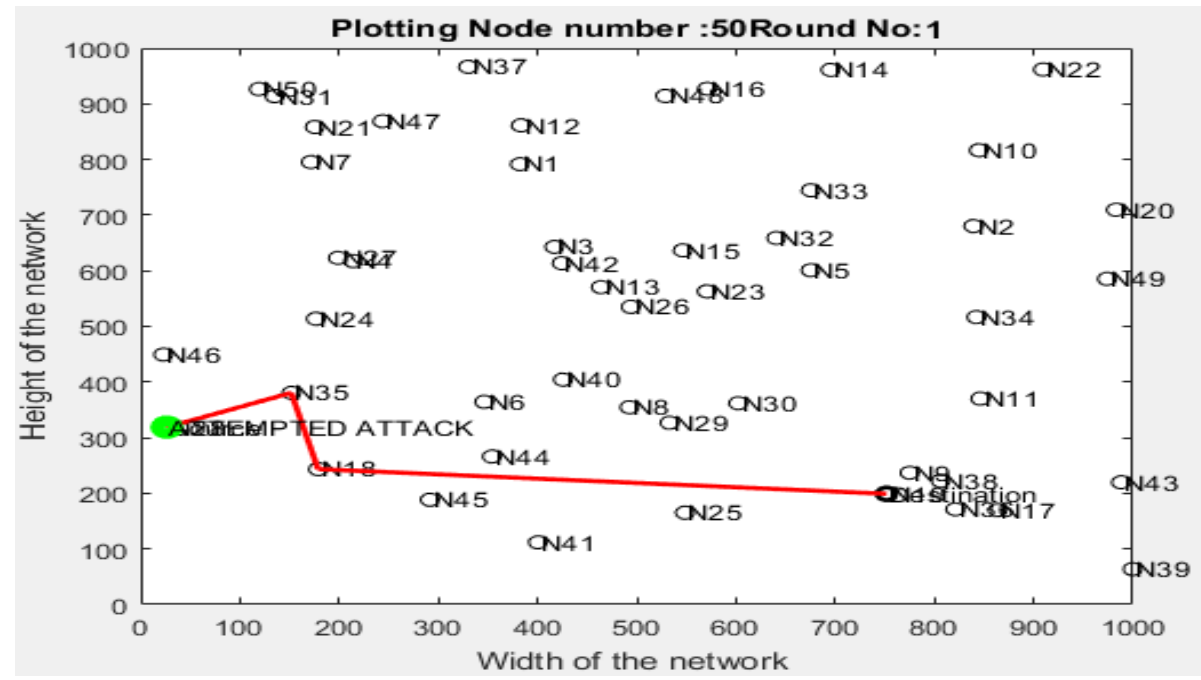

Figure 7: Route between source and destination

Above figure is describing the route between the source and the destination by using the routing protocol. The route is being used for transferring the data.

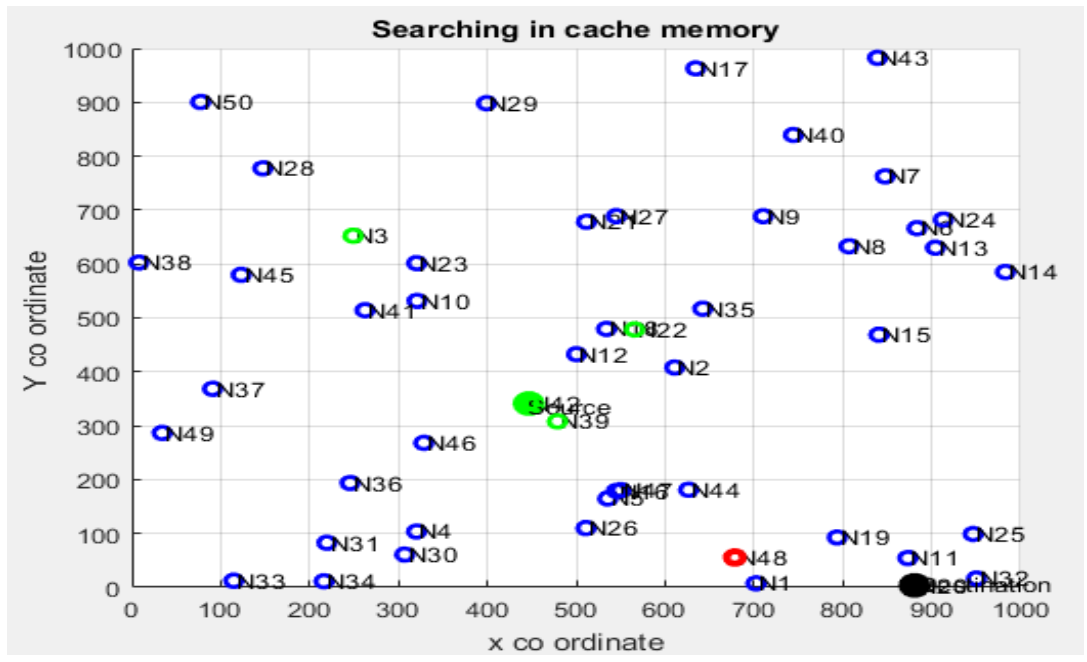

Figure 8: Node route

Above figure is depicting the route of the source nodes to

the black circle and the green circle is depicting the source the destination nodes. The destination nodes are depicted by nodes.

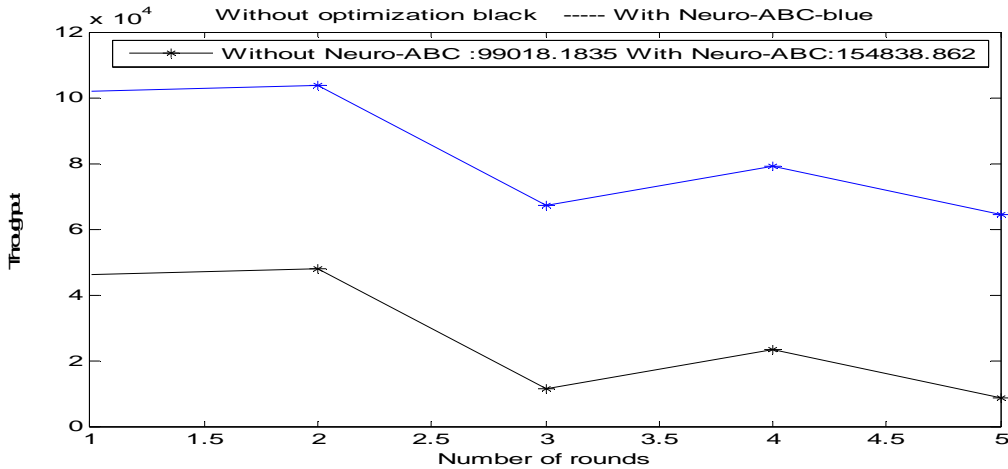

Figure 9: Throughput without optimization and with neuro-ABC 
The variability in the network by means of throughput is depicted in above figure. Numbers of rounds taken to execute the work are shown in $\mathrm{X}$-axis and values obtained after the execution of throughout are shown in Y-axis. For 5 rounds, without optimization, throughput is 99018.1835 and with optimization, it is 154837.862 .

Table 1: Throughput without optimization and with Neuro-ABC

\begin{tabular}{|l|l|l|l|}
\hline Number of rounds & $\begin{array}{l}\text { Throughput without } \\
\text { optimization }\left(10^{4}\right)\end{array}$ & $\begin{array}{l}\text { Throughput with Neuro- } \\
\text { ABC }\left(10^{4}\right)\end{array}$ & Average $\left(10^{4}\right)$ \\
\cline { 1 - 3 } 3 & 4.5 & 10.5 & \\
\hline 2 & 5 & 11 & \\
\hline 3 & 2 & 7 & \\
\hline 4 & 3 & 9 & \\
\hline 5 & 1 & 7 & \\
\hline
\end{tabular}

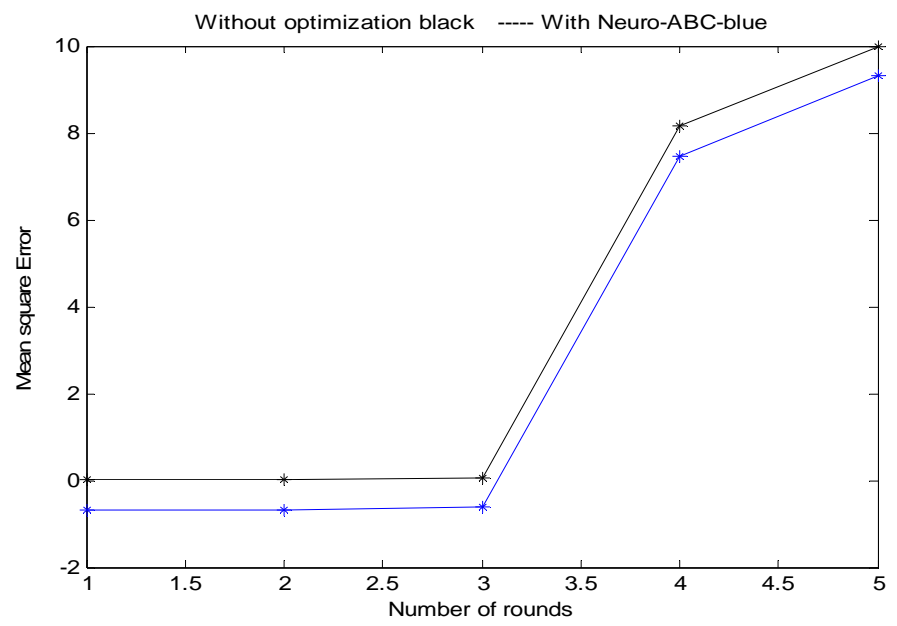

Figure 10: Mean Square Error with optimization and with Neuro-ABC

Above figure defines the result obtained after the evaluation of the simulation work. The variability in the network by means of MSE is depicted in above figure. Numbers of rounds taken to execute the work are shown in X-axis and values obtained after the execution of MSE are shown in Yaxis.

Table 2: Mean Square error with and without optimization

\begin{tabular}{|l|l|l|l|}
\hline Number of round & $\begin{array}{l}\text { Mean Square Error } \\
\text { without optimization }\end{array}$ & MSE with Neuro-ABC & Average \\
\hline 1 & 0 & -0.5 & \multirow{3}{*}{$3.7,2.9$} \\
\hline 2 & 0 & -0.5 & \\
\hline 3 & 0.5 & -0.5 & \\
\hline 4 & 8 & 7 & \\
\hline 5 & 10 & 9 & \\
\hline
\end{tabular}


From the above table values obtained we are concluding that the average value of MSE without optimization is 3.7 and with optimization it get reduced and become 2.9.

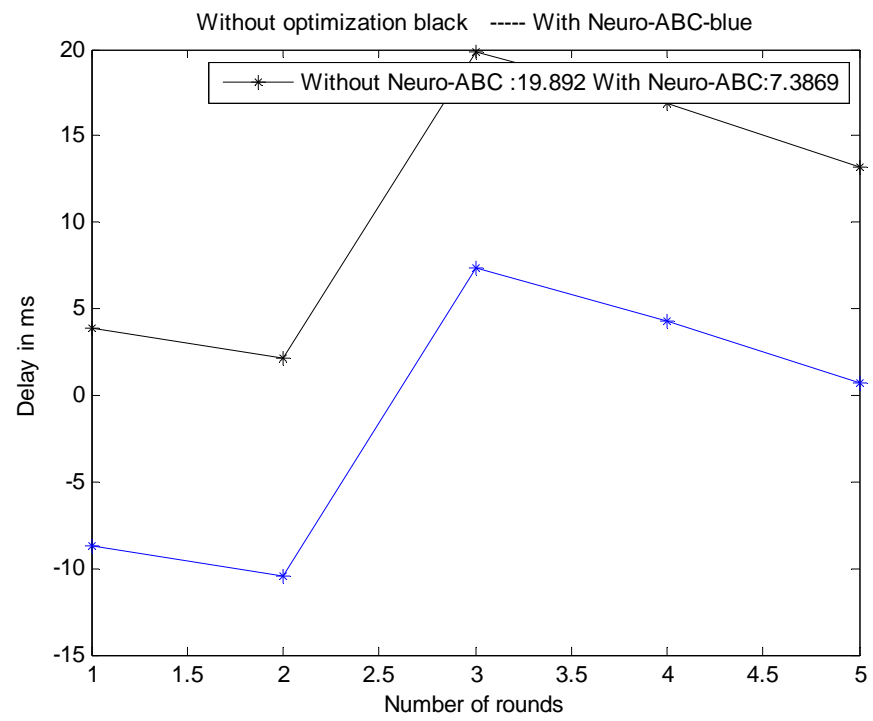

Figure 11: Delay with and without optimization

Above figure defines the result obtained after the evaluation of the simulation work. The variability in the network by means of Delay is depicted in above figure. Numbers of rounds taken to execute the work are shown in X-axis and values obtained after the execution of delay are shown in $\mathrm{Y}$ axis.
Negative value of delay is obtained for proposed work whereas when usage of $\mathrm{NN}$ is used with $\mathrm{ABC}$, delay is 11.4msec approximately.

Table 3: Delay values for without and with optimization

\begin{tabular}{|l|l|l|l|}
\hline Number of round & $\begin{array}{l}\text { Delay without } \\
\text { optimization }\end{array}$ & Delay with Neuro-ABC & \multirow{2}{*}{ Average } \\
\cline { 1 - 3 } 1 & 4 & -8 & \multirow{3}{*}{$1.4,-1.6$} \\
\cline { 1 - 3 } & 2 & -10 & \\
\hline 3 & 20 & 5 & \\
\cline { 1 - 3 } 5 & 18 & 4 & 1 \\
\hline
\end{tabular}




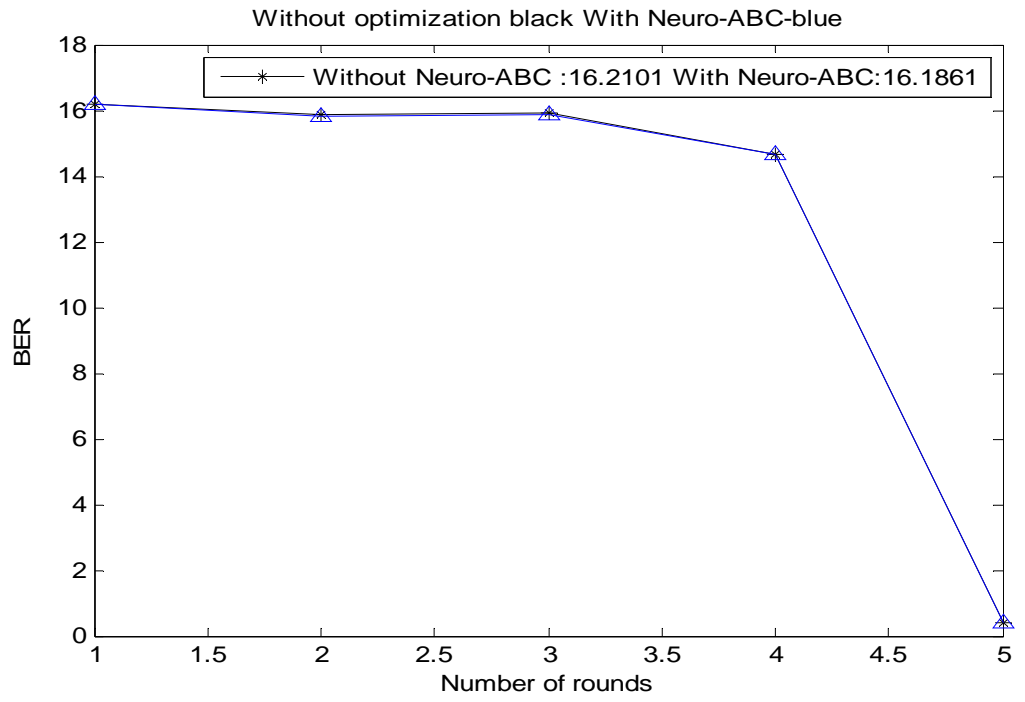

Figure 12: BER with and without optimization

Above figure defines the result obtained after the evaluation of the simulation work. The variability in the network by means of BER is depicted in above figure. Numbers of rounds taken to execute the work are shown in $\mathrm{X}$-axis and values obtained after the execution of BER are shown in Yaxis.
16.2101 is the value when no optimization algorithm is used and when usage of $\mathrm{ABC}$ and $\mathrm{NN}$ is used, average value of BER is 18.1861.It means there is reduction in the values of BER when the usage of optimization algorithm is considered.

Table 4: BER with and without optimization

\begin{tabular}{|c|c|c|c|}
\hline Number of round & $\begin{array}{l}\text { BER } \\
\text { optimization }\end{array}$ & BER with Neuro-ABC & Average \\
\hline 1 & 16.01 & 16 & \multirow{5}{*}{$16.21,16.18$} \\
\hline 2 & 16 & 15.98 & \\
\hline 3 & 16.02 & 16 & \\
\hline 4 & 15.87 & 15.86 & \\
\hline 5 & 0.89 & 0.88 & \\
\hline
\end{tabular}

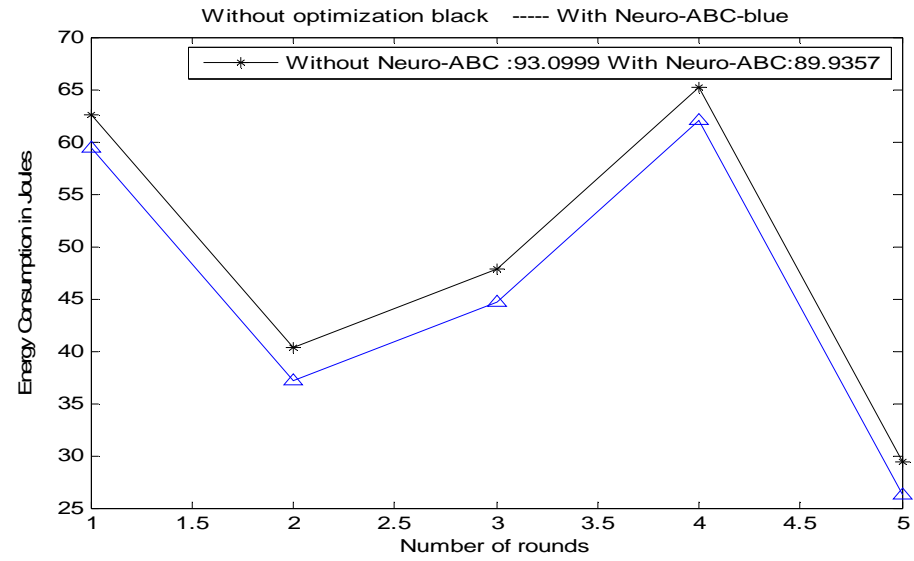

Figure 13: Energy consumption with and without optimization

Above figure defines the result obtained after the evaluation of the simulation work. The variability in the network by means of energy consumption is depicted in above figure. Numbers of rounds taken to execute the work are shown in (C) 2015-19, IJARCS All Rights Reserved
$\mathrm{X}$-axis and values obtained after the execution of energy consumption are shown in $\mathrm{Y}$-axis. 
93.0999 is the value when no optimization algorithm is used and when usage of $\mathrm{ABC}$ and $\mathrm{NN}$ is used, average value of energy consumption is 89.9357.It means there is reduction in the values of energy consumption when the usage of optimization algorithm is considered. Energy consumption should be less for the reliability of the system.

Table Error! No text of specified style in document.: Energy consumption values for with and without optimization

\begin{tabular}{|c|c|c|c|}
\hline Number of round & $\begin{array}{l}\text { Energy Consumption } \\
\text { without optimization }\end{array}$ & $\begin{array}{l}\text { Energy Consumption } \\
\text { with Neuro-ABC }\end{array}$ & Average \\
\hline 1 & 63 & 60 & \multirow{5}{*}{$50,45.8$} \\
\hline 2 & 42 & 35 & \\
\hline 3 & 50 & 45 & \\
\hline 4 & 65 & 63 & \\
\hline 5 & 30 & 26 & \\
\hline
\end{tabular}

\section{CONCLUSION}

MANET is known as the mostly utilized network because of its mobile character. Though, these network can be affected from sink hole attack. When the attack comes into the network, the network Performance degraded. In this research, usage of $\mathrm{ABC}$ and $\mathrm{ANN}$ Is taken place for the mitigation of sink hole attack in the network. ABC has been used for the optimization whereas ANN Is used as a classification method. OLSR routing protocol is used for discovering the route among source and destination. Simulation has been conducted on MATLAB tool with 100 nodes approximately and for 5 rounds. The performance has been checked on the basis of various parameters, viz. Throughput, BER, energy consumption and MSE. The results are calculated on the basis of with and without optimization strategy to show the effectives of the work. It is concluded of the rate of energy consumption, BER is reduced and rate of throughout is enhanced when usage of optimization algorithm is taken place

Future lies in using other routing protocols, like DSR, AODV, and TORA and so on. For optimization different algorithms like Genetic algorithm (GA) and particle swarm optimization (PSO) will be used. For classification algorithms like Fuzzy logic and Support vector machine (SVM) will be used.

\section{REFERENCES}

[1] Loo, J., Mauri, J. L., \& Ortiz, J. H. (Eds.). (2016). Mobile ad hoc networks: current status and future trends. CRC Press.

[2] Conti, M., \& Giordano, S. (2014). Mobile ad hoc networking: milestones, challenges, and new research directions. IEEE Communications Magazine, 52(1), 8596.

[3] Wei, Z., Tang, H., Yu, F. R., Wang, M., \& Mason, P. (2014). Security enhancements for mobile ad hoc networks with trust management using uncertain reasoning. IEEE Transactions on Vehicular Technology, 63(9), 4647-4658.

[4] Pathan, A. S. K. (Ed.). (2016). Security of self-organizing networks: MANET, WSN, WMN, VANET. CRC press.

[5] Darwish, S. (2015). A simulation-based methodology for the assessment of server-based security architectures for mobile ad hoc networks (MANETs) (Doctoral dissertation, Brunel University London).

[6] Arora, S. K., Puri, N., \& Sharma, S. (2015). Performance analysis and detection and correction of sink hole attack in MANET. International Journal of Applied Engineering and research, 10(1), 12133-12144.

[7] Singh, A., \& Singh, T. (2016). Review on Detection and Prevention of Sink Hole Attack In network. Global Journal of Computers \& Technology, 5(2), 289-292.

[8] Sharma, S., \& Jaswak, K. B. (2017). Mitigation of Sinkhole Attack in MANET Using ACO.

[9] Singh, T. (2017). DESIGN AND ANALYSIS OF ADHOC ON DEMAND DISTANCE VECTOR ROUTING (AODV) IN WIRELESS SENSOR NETWORK USING NEURAL NETWORK. Global Journal of Computers \& Technology, 6(1), 344-353.

[10] Hinton, G., Vinyals, O., \& Dea Karaboga, D., Gorkemli, B., Ozturk, C., \& Karaboga, N. (2014). A comprehensive survey: artificial bee colony (ABC) algorithm and applications. Artificial Intelligence Review, 42(1), 21-57.

[11] .02531.

[12] Kıran, M. S., \& Fındık, O. (2015). A directed artificial bee colony algorithm. Applied Soft Computing, 26, 454-462.

[13] Murthy, M. S. N., Sriram, G. G., \& Abhiram, B. (2016). Enhanced bee colony optimization mechanism in content recommendation system.

[14] Agrwal, S. L., Khandelwal, R., Sharma, P., \& Gupta, S. K. (2016, October). Analysis of detection algorithm of Sinkhole attack \& QoS on AODV for MANET. In Next Generation Computing Technologies (NGCT), 2016 2nd International Conference on (pp. 839-842). IEEE.

[15] Tajinder deep singh \& Harpreet kaur arora (2013). Detection and correction of sinkhole attack with novel method in wireless censor network using NS2 tools in international generals of computer science and applied volume 4 number 2 